\title{
Martensitic Transformation in Ni-Mn-Ga Alloy Under High Magnetic Fields
}

\author{
Volodymyr A. Chernenko ${ }^{1,6}$, Victor A. Lvov ${ }^{2}$, Takeshi Kanomata ${ }^{3}$, Tomoyuki Kakeshita ${ }^{4}$, \\ Keiichi Koyama ${ }^{5}$ and Stefano Besseghini ${ }^{6}$ \\ ${ }^{1}$ Institute of Magnetism, Kyiv 03142, Ukraine \\ ${ }^{2}$ Department of Radiophysics, Taras Shevchenko University, Kyiv 03022, Ukraine \\ ${ }^{3}$ Department of Applied Physics, Faculty of Engineering, Tohoku Gakuin University, Tagajo 985-8537, Japan \\ ${ }^{4}$ Department of Materials Science and Engineering, Graduate School of Engineering, Osaka University, Suita 565-0871, Japan \\ ${ }^{5}$ Institute for Materials Research, Tohoku University, Sendai 980-8577, Japan \\ ${ }^{6}$ CNR-IENI, Lecco 23900, Italy
}

\begin{abstract}
An effect of magnetic field on the martensitic transformation $(M T)$ temperature in ferromagnetic shape memory $\mathrm{Ni}_{49.4} \mathrm{Mn}_{27.7} \mathrm{Ga}_{22.9}$ single crystal with the $M T$ temperature $285 \mathrm{~K}$ is studied by measuring and theoretical treatment of the magnetization versus temperature dependencies in a wide range of the high magnetic fields higher than saturating one. In this way, a linear approximation of the field dependence of $M T$ temperature was proved for the high-field range. The linear increase of transformation temperature is characterized by the slope of $3.5 \times 10^{-2} \mathrm{~K} / \mathrm{kOe}$. This value is essentially larger than the value $2 \times 10^{-2} \mathrm{~K} / \mathrm{kOe}$ reported by Gonzales-Comas et al. [Phys. Rev. B 60 (1999) 7085-7090] for the $\mathrm{Ni}_{49.5} \mathrm{Mn}_{25.4} \mathrm{Ga}_{25.1}$ alloy with a low $M T$ temperature. This difference is in an agreement with the predictions of the Landau theory.
\end{abstract}

(Received October 6, 2005; Accepted January 25, 2006; Published March 15, 2006)

Keywords: ferromagnetic shape-memory alloy, martensitic transformation, high magnetic field, Landau theory

\section{Introduction}

The stoichiometric and off-stoichiometric Heusler $\mathrm{Ni}_{2}$ MnGa alloys exhibiting martensitic transformation combine a thermoelastic behavior and ferromagnetic ordering whereby giving rise to unusual magnetic and magnetoelastic properties (see Refs. 1, 2) and references therein). A multifunctional ability of these alloys and other similar systems such as $\mathrm{Fe}_{3} \mathrm{Pt}$ and $\mathrm{Fe}-\mathrm{Pd}^{3)}$ is very promising for the applications. Among the interesting physical phenomena related to the practically important $\mathrm{Ni}-\mathrm{Mn}-\mathrm{Ga}$ alloys transforming martensitically below Curie temperature, $T_{\mathrm{C}}$, are numerous magnetic anomalies which accompany the spontaneous deformation of the parent cubic lattice in the course of $M T$. In particular, a jump of spontaneous magnetization, $\Delta M$, is worth noting. ${ }^{4-6)}$ For the $M T$ 's with a small volume effect, this jump is the main thermodynamic reason of magnetic field influence on the transformation temperature, $T_{\mathrm{M}}{ }^{7,8)}$ In Ni-Mn-Ga alloys, the value of magnetization jump is found to be dependent on the temperature interval between the $T_{\mathrm{M}}$ and $T_{\mathrm{C}} \cdot{ }^{6,9,10)}$ This dependence was recently studied in a systematic way for the Ni-rich/Mn-deficient alloy series ${ }^{6)}$ Normally, the experimental determination of the $\Delta M$ is performed by recording the temperature dependence of magnetization, $M(T)$, in the saturating magnetic field, $H_{\mathrm{S}}$, which is in case of $\mathrm{Ni}-\mathrm{Mn}-\mathrm{Ga}$ alloys is around $10 \mathrm{kOe}$. As far as the jump-like anomaly on $M(T)$ curve is smeared and can be field-dependent, the correct determination of both the $\Delta M$ value and $T_{\mathrm{M}}(H)$ function is difficult. In present work, we suggest an approach allowing an unequivocal interpretation and reliable reading of aforementioned characteristics from $M(T)$ curves. The experimental investigation of the magnetic field influence on the $M T$ in single crystalline Mn-rich alloy $\mathrm{Ni}_{49.4} \mathrm{Mn}_{27.7} \mathrm{Ga}_{22.9}$ is performed. The results are treated in the framework of suggested approach which is based on Landau theory.

\section{Experiment}

A single crystal was grown by the Bridgman method from an induction melted and cast ingot of Ni-Mn-Ga alloy. The high purity elements, $99.99 \%$ for $\mathrm{Ni}$ and $\mathrm{Mn}$ and $99.9999 \%$ for $\mathrm{Ga}$, were used for alloy preparation. According to X-ray spectral analysis, the composition of single crystal was determined to be $\mathrm{Ni}_{49.4} \mathrm{Mn}_{27.7} \mathrm{Ga}_{22.9}$ with uncertainty of \pm 0.3 at $\%$. The crystal was annealed at $1170 \mathrm{~K}$ for $27 \mathrm{~h}$ in argon atmosphere followed by slow cooling. The orientation of the crystal was determined by Laue X-ray back reflection method. Differential scanning calorimetry (DSC) and temperature ramps of low field ac magnetic permeability were used to determine routinely the characteristic temperatures of $M T$ as well as Curie temperature, $T_{\mathrm{C}}$. The $M T$ with $T_{\mathrm{M}} \approx$ $285 \mathrm{~K}$ and temperature hysteresis of $8 \mathrm{~K}$ together with $T_{\mathrm{C}} \approx$ $385 \mathrm{~K}$ were determined from these measurements. Electron diffraction patterns of in-situ cooled thin foil show 4 not equally spaced extra spots dividing the distance between the fundamental reflections along $\langle 110\rangle^{*}$ directions of initial cubic phase, evidencing that 5-layered tetragonal martensitic phase is formed. A spontaneous deformation arising in the course of $M T$ is characterized by the tetragonality ratio $c / a \approx$ 0.94 , where $a$ and $c$ are the lattice parameters of the tetragonal unit cell.

The magnetization behavior was studied by a Quantum Design SQUID magnetometer. The specimen size was $0.6 \times$ $0.6 \times 3 \mathrm{~mm}^{3}$ with long edge oriented along the [001] crystallographic direction. The magnetic field was applied along long axis of the specimen. The measurements were performed under constant magnetic fields up to $70 \mathrm{kOe}$ during slow heating $(0.5 \mathrm{~K} / \mathrm{min})$ in the temperature range of 240-320 K. The results are depicted in Fig. 1. Each curve clearly show a jump accompanying the $M T$ followed by a strong descending behavior due to approaching to the Curie temperature. A different sign of jumps is well known ${ }^{2,6,9)}$ to 


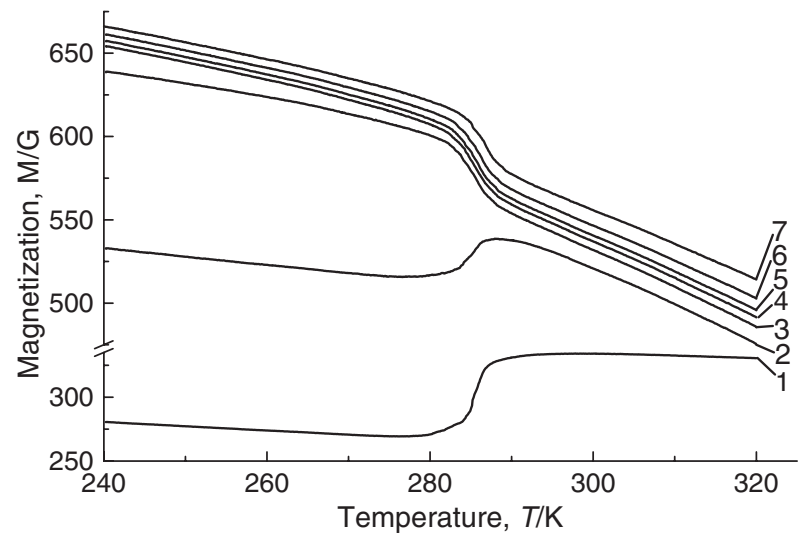

Fig. 1 Temperature dependencies of magnetization showing jumps at $M T$. Measurements are made at different constant magnetic fields, kOe: (1) -1.0 ; (2) -5.0 ; (3) -10.0 ; (4) -15.0 ; (5) -20.0 ; (6) -30.0 ; and (7) -50.0 .

occur below and above saturating field $H_{\mathrm{S}} \geq 10 \mathrm{kOe}$. The experimental data in Fig. 1 obtained at the fields above $H_{\mathrm{s}}$ are treated in Sec. 3 using the Landau approach for the description of the cubic-tetragonal $M T$ in which the anisotropic terms are naturally disregarded. In this analysis, our previous results of Refs. 5, 9, 11) will be taken into account.

\section{Theoretical analysis of the experimental results}

\subsection{Jump of magnetization at the transformation tem- perature}

Let $T_{\mathrm{s}}$ and $T_{\mathrm{f}}$ be the temperatures of the start and finish of $M T$, respectively. According to Refs. 5, 9, 11) the shift $\Delta T_{\mathrm{M}}(H)$ of the martensitic transformation temperature $T_{\mathrm{M}}=$ $\left(T_{\mathrm{s}}+T_{\mathrm{f}}\right) / 2$ in the $\mathrm{Ni}-\mathrm{Mn}-\mathrm{Ga}$ alloy affected by the strong magnetic field $H$ is caused mainly by the jump of magnetization value $\Delta M \equiv M\left(T_{\mathrm{s}}\right)-M\left(T_{\mathrm{f}}\right)$ in the narrow temperature range corresponding to the $M T$ temperature. To compute the $\Delta M$ value, the temperature dependence of magnetization should be considered and used subsequently for the theoretical determination of $\Delta T_{\mathrm{M}}(H)$ function.

For the Ni-Mn-Ga single crystalline specimen affected by the magnetic field $\boldsymbol{H} \|[001]$ exceeding in magnitude a saturation value $H_{\mathrm{S}}$, the magnetization vector $\boldsymbol{M}$ is parallel to [001]. As far as the direction of this vector is fixed, the temperature dependence of its modulus can be derived from the following expression for the Landau potential:

$$
F=3 C^{\prime}\left(u_{2}^{2}+u_{3}^{2}\right) / 2+J(T) y^{2} / 2-\delta_{0} y^{2} u_{1}-H M(0) y,
$$

where

$$
\begin{aligned}
& y=M(T) / M(0), \\
& u_{1}=\left(\varepsilon_{x x}+\varepsilon_{y y}+\varepsilon_{z z}\right) / 3, \\
& u_{2}=\sqrt{3}\left(\varepsilon_{x x}-\varepsilon_{y y}\right), \quad u_{3}=2 \varepsilon_{z z}-\varepsilon_{y y}-\varepsilon_{x x},
\end{aligned}
$$

$\varepsilon_{i i}$ are the components of strain tensor, $C^{\prime}$ is shear modulus, $J(T)$ is the spin exchange parameter, $\delta_{0}$ is a magnetoelastic constant responsible for the spontaneous volume magnetostriction of the crystal. The temperature dependence of the exchange parameter $J(T)$ can be presented in the vicinity of Curie temperature $T_{\mathrm{C}} \equiv T_{\mathrm{CA}}$ in a standard form $J(T)=$
$\zeta\left(T-T_{\mathrm{CA}}\right) / T_{\mathrm{CA}}$, where $\zeta$ is a proportionality coefficient.

It was demonstrated ${ }^{5,11)}$ that the experimental temperature dependencies of the magnetization values of three different types of Ni-Mn-Ga alloys are satisfactorily described by the function

$$
M(H, T)=M(H, 0) y(T),
$$

where $y(T)$ satisfies the equation

$$
y(T)=\tanh \left[\left(T_{\mathrm{CA}} / T\right) y(T)\right]
$$

when $T>T_{\mathrm{M}}$ and should be found from the equation

$$
y(T)=\tanh \left[\left(T_{\mathrm{CM}} / T\right) y(T)\right]
$$

when $T<T_{\mathrm{M}}$. The characteristic temperatures $T_{\mathrm{CA}}$ and $T_{\mathrm{CM}}$ are the Curie temperatures of the austenitic and martensitic phases, respectively. These temperatures were introduced in Ref. 12). The solution of the eq. (4) will be denoted below as $y_{\mathrm{A}}(T)$ while the function satisfying the eq. (5) will be referred to as $y_{\mathrm{M}}(T)$. For the quasistoichiometric Ni-Mn-Ga alloys, the $M T$ temperature is lower than the Curie temperature, and therefore, the temperature $T_{\mathrm{CA}}$ is the real Curie temperature of these alloys (as indicated above) while $T_{\mathrm{CM}}$ is a parameter controlling the temperature dependence of magnetization below $M T$ temperature.

A comparison of the theoretical temperature dependencies of magnetization with the experimental ones indicates that the difference between the characteristic temperatures $T_{\mathrm{CA}}$ and $T_{\mathrm{CM}}$ arises mainly from the difference in the intensity of spin exchange interaction in the austenitic and martensitic phases. ${ }^{5,11)}$ In turn, the difference in spin exchange is caused by the jump-like volume variation $\Delta V / V$ accompanying the $M T{ }^{5,11)}$ Thus, the difference in characteristic temperatures is proportional to both magnetoelastic parameter $\delta_{0}$ and equilibrium value of $u_{1}$ variable, because $u_{1}=(1 / 3)(\Delta V / V)$, for more details see Ref. 5). Therefore the "virtual" temperature $T_{\mathrm{CM}}$ can be expressed through the observable temperature $T_{\mathrm{CA}}$ as

$$
T_{\mathrm{CM}}=T_{\mathrm{CA}}\left[1+2\left(\delta_{0} / \zeta\right) u_{1}\right]
$$

The alloy studied in this work has $T_{\mathrm{CA}} \approx 385 \mathrm{~K}$. Moreover, the values $\delta_{0} \approx-0.4 \mathrm{GPa}, \zeta \approx 0.1 \mathrm{GPa}, u_{1} \sim-10^{-2}$ are inherent for the different types of Ni-Mn-Ga alloys ${ }^{5)}$ so, they are assumed to be applicable for our alloy. All just-mentioned values result in the estimation $T_{\mathrm{CM}}-T_{\mathrm{CA}} \approx 30 \mathrm{~K}$. Figure 2 shows a theoretical temperature dependence of magnetization computed for the $\mathrm{Ni}_{49.4} \mathrm{Mn}_{27.7} \mathrm{Ga}_{22.9}$ martensite with $T_{\mathrm{M}} \approx 285 \mathrm{~K}$. The notations used are shown in Fig. 2 as well.

The eqs. (3)-(6) enable the computation of $\Delta M$ value. This value can also be presented in more explicit form when the $M T$ temperature of the specimen is far enough from the characteristic temperature $T_{\mathrm{CA}}$. In this case, the functions computed from the eqs. (4) and (5) are close to each other [i.e., $\left.y_{\mathrm{M}}(T)-y_{\mathrm{A}}(T) \ll y_{\mathrm{A}}(T)\right]$. The linearization of the eq. (5) in small parameter $\eta=2\left(\delta_{0} / \zeta\right) u_{1}$ results in the approximate expression

$$
\Delta M=\eta M(H, 0) \cdot \frac{y_{\mathrm{A}}\left(T_{\mathrm{M}}\right)\left[1-y_{\mathrm{A}}^{2}\left(T_{\mathrm{M}}\right)\right]}{\left(T_{\mathrm{M}} / T_{\mathrm{CA}}\right)-\left[1-y_{\mathrm{A}}^{2}\left(T_{\mathrm{M}}\right)\right]}
$$

The values $\eta \approx 0.08$ and $M(H, 0) \approx 700 \mathrm{G}$ are approximately universal for the different alloys belonging to the $\mathrm{Ni}-$ 


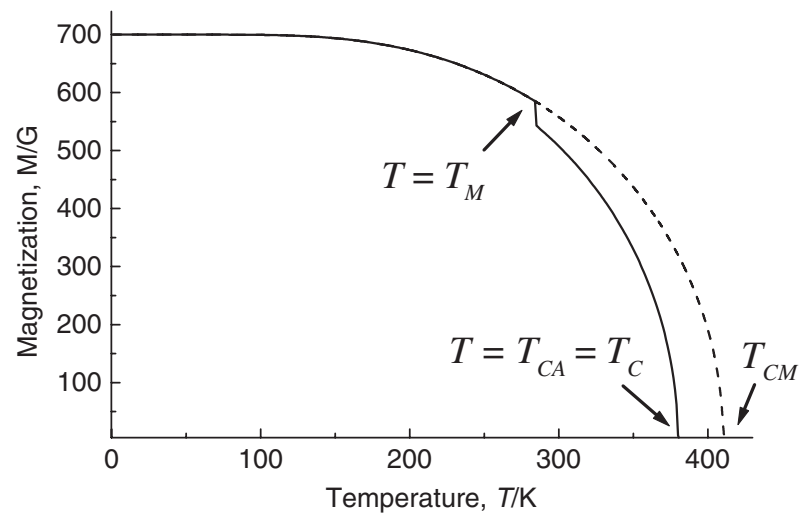

Fig. 2 Magnetization versus temperature dependence for $\mathrm{Ni}_{49.4} \mathrm{Mn}_{27.7-}$ $\mathrm{Ga}_{22.9}$ computed using the eqs. (3)-(6).

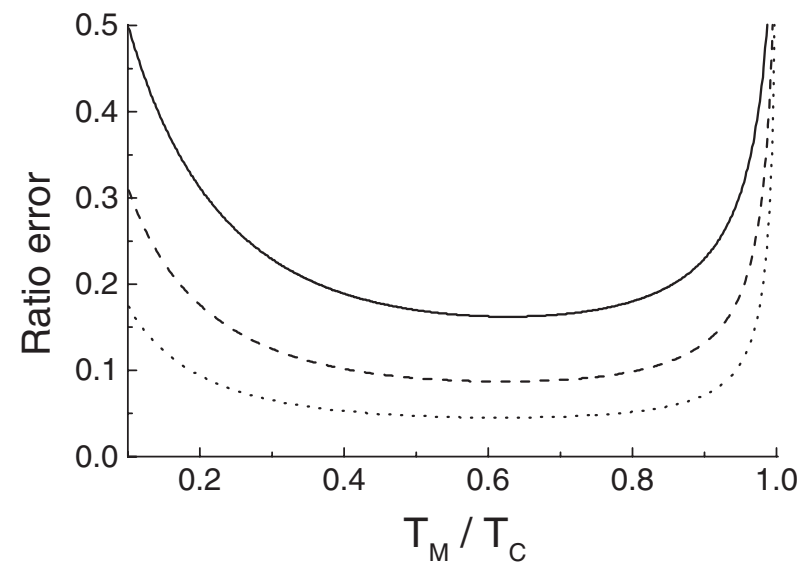

Fig. 3 The ratio error of the theoretical determination of $\Delta M$ value found from the approximate eq. (7) with $\eta$ equal to 0.02 (dots), 0.04 (dashes) and 0.08 (solid line).

Mn-Ga system. ${ }^{5)}$ Thus, the jump of magnetization in the vicinity of $M T$ temperature can be approximately evaluated from the eq. (7) using the numerical solution of the standard eq. (4). The ratio error in $\Delta M$ value arising when the approximate eqs. (4) and (7) are used instead of exact eqs. (4) and (5) is shown in Fig. 3. This figure demonstrates, that the eq. (7) provides a satisfactory majorant estimation of magnetization jump in the practically important temperature range $0.4<T_{\mathrm{M}} / T_{\mathrm{C}}<0.8$.

For the studied alloy with $T_{\mathrm{CA}} \approx 385 \mathrm{~K}$ and $T_{\mathrm{M}} \approx 285 \mathrm{~K}$, the approximate relationships eqs. (4) and (7) result in the value $\Delta M=46 \mathrm{G}$ while the value computed from the eqs. (3)-(5) is equal to $39 \mathrm{G}$. Experimental values obtained from the $M(T)$ curves measured for the different magnitudes of magnetic field are presented in the Table 1. The handling procedure of the experimental curves is illustrated in Fig. 4.

The jumps of magnetization observed in the fields equal to 20 and $30 \mathrm{kOe}$ are close in value to both theoretical estimations reported above. The magnetization jump observed in the field equal to $10 \mathrm{kOe}$ is smaller because this field value is not sufficient for the complete magnetic saturation of the martensitic phase. The reduction of $\Delta M$ value in the field equal to $50 \mathrm{kOe}$ can be assumedly related to the difference in the magnetic susceptibilities of
Table 1 The $\Delta M$ values determined from the experimental dependencies $M(T)$ and model equations for the $\mathrm{Ni}_{49.4} \mathrm{Mn}_{27.7} \mathrm{Ga}_{22.9}$ alloy.

\begin{tabular}{ccccc}
\hline$H(\mathrm{kOe})$ & 10 & 20 & 30 & 50 \\
\hline$\Delta M(G)$, eqs. (4), (7) & 46 & 46 & 46 & 46 \\
$\Delta M(G)$, eqs. (3)-(5) & 39 & 39 & 39 & 39 \\
$\Delta M(G)$, experiment & 34 & 43 & 42 & 37 \\
\hline
\end{tabular}

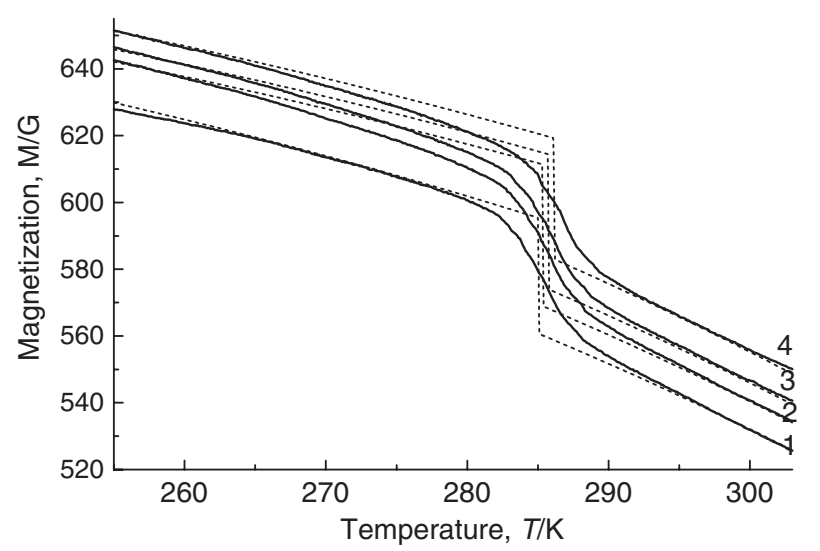

Fig. 4 Graphic determination of the experimental values of the jumps of magnetization for the $\mathrm{Ni}_{49.4} \mathrm{Mn}_{27.7} \mathrm{Ga}_{22.9}$ alloy: solid lines present the experimental temperature dependencies of magnetization taken for the magnetic field values $10 \mathrm{kOe}(1), 20 \mathrm{kOe}(2), 30 \mathrm{kOe}(3)$, and $50 \mathrm{kOe}(4)$; the dashed tangential lines are fitted to the experimental curves.

austenitic and martensitic phases. It should be stressed, that some discrepancy between the experimental and theoretical values may be also caused by the tentative character of the graphical procedure presented in Fig. 4.

\subsection{Field dependence of transformation temperature}

The physical mechanisms responsible for the magnetic field dependence of $M T$ temperature were considered in Ref. 7-9) and overview. ${ }^{13)}$ It was shown recently that due to the small value of volume change accompanying $M T$ in $\mathrm{Ni}-$ $\mathrm{Mn}-\mathrm{Ga}$ alloys the shift of $M T$ temperature in the strong magnetic field can be evaluated from the following expression: ${ }^{9)}$

$$
\begin{aligned}
\Delta T_{\mathrm{M}} & \equiv T_{\mathrm{M}}(H)-T_{\mathrm{M}}(0) \\
& =(3 / 4) H \Delta M\left(d C^{\prime} / d T\right)^{-1}(1-c / a)^{-2},
\end{aligned}
$$

where $d C^{\prime} / d T=43 \mathrm{MPa} / \mathrm{K}^{14)}$ The linear functions $\Delta T_{\mathrm{M}}(H)$ computed from the eq. (8) are presented in the Fig. 5 where the solid line corresponds to the exact solutions $y_{\mathrm{A}}(T)$ and $y_{\mathrm{M}}(T)$ of the eqs. (4) and (5), respectively, while the dashed line is plotted using the approximate eq. (7). A good agreement between the theoretical and experimental results takes place in the field range above the saturating field value, where the free energy expansion eq. (1) is valid. In contrast to the high field range, the non-monotonous dependence of MT temperature versus magnetic field is observed experimentally below saturating field value $H_{\mathrm{s}} \approx 10-12 \mathrm{kOe}$. A similar result was also reported in Ref. 15). In particular, the initial decrease of $T_{\mathrm{M}}$ as a function of field was tentatively explained by the different sign of $\Delta M$ at non-saturating and saturating magnetic fields. ${ }^{15}$ ) Such a behavior should be clarified in the forthcoming studies. 


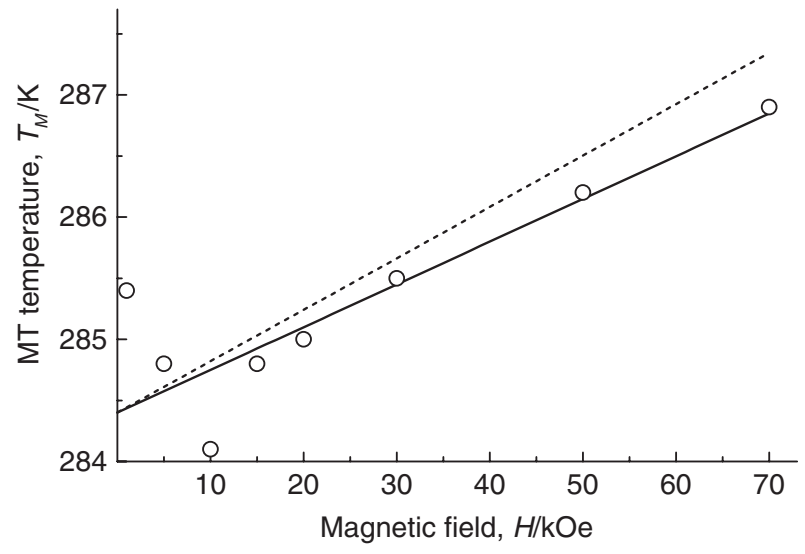

Fig. 5 Martensitic transformation temperature versus magnetic field: experiment (circles) and theory (straight lines). The solid line is computed from the eqs. (3)-(5), the dashed line is plotted using the approximate eqs. (4) and (7).

\section{Conclusions}

A series of magnetization versus temperature dependencies was measured for the ferromagnetic $\mathrm{Ni}_{49.4} \mathrm{Mn}_{27.7^{-}}$ $\mathrm{Ga}_{22.9}$ single crystalline alloy in a wide range of magnetic fields. These measurements enabled the experimental determination of magnetization jump accompanying $M T$ together with a related shift of $M T$ temperature as a function of the magnetic field. The experimental dependence of $M T$ temperature on the applied magnetic field higher than saturating one turned out to be in a good agreement with the linear function computed in the framework of Landau approach to the description of the cubic-tetragonal martensitic transformation in the ferromagnetic shape memory alloy. This dependence is characterized by the slope of $3.5 \times 10^{-2} \mathrm{~K} / \mathrm{kOe}$. This slope ratio is notably larger than the value $2 \times 10^{-2} \mathrm{~K} / \mathrm{kOe}$ reported in Ref. 4) for the $\mathrm{Ni}_{49.5} \mathrm{Mn}_{25.4} \mathrm{Ga}_{25.1}$ alloy with the low $M T$ temperature. A comparatively small value of $d T_{\mathrm{M}} / d H$ obtained for the low temperature alloy correlates with the eqs. (7) and (8) which demonstrate the reduction of $\Delta M$ and $\Delta T_{\mathrm{M}}$ values as a function of the diminishing $M T$ temperature.

\section{Acknowledgements}

VAC is grateful to Tohoku Gakuin University for financing his stay at Mechanical Engineering Department. The help of D. Kikuchi in measurements is greatly acknowledged.

\section{REFERENCES}

1) V. A. Chernenko, E. Cesari, V. V. Kokorin and I. N. Vitenko: Scr. Met. et Mat. 33 (1995) 1239-1244.

2) A. N. Vasil'ev, V. D. Buchel'nikov, T. Takagi, V. V. Khovailo and E. A. Estrin: Physics-Uspekhi 46 (2003) 559-588.

3) T. Kakeshita and K.Ullakko: MRS Bulletin 27 (2002) 105-108.

4) A. Gonzàlez-Comas, E. Obradó, Ll. Manosa, A. Planes, V. A. Chernenko, B. Jan Hattink and A. Labarta: Phys. Rev. B 60 (1999) 7085-7090.

5) V. A. Chernenko, V. A. L'vov, S. P. Zagorodnyuk and T. Takagi: Phys. Rev. B 67 (2003) 064407.

6) V. V. Khovailo, V. Novosad, T. Takagi, D. A. Filippov, R. Z. Levitin and A. N. Vasil'ev: Phys. Rev. B 70 (2004) 174413.

7) M. A. Krivoglaz and V. D. Sadovsky: Fiz. Metal. Metalloved. 18 (1964) 502-504.

8) K. Shimizu, N. Yamano, T. Kakeshita, M. Ono, K. Sugiyama and M. Date: Mater. Sci. Forum 56-58 (1990) 235-238.

9) V. A. Chernenko, O. Babii, V. A. Lvov and P. G. McCormick: Mater. Sci. Forum 327-328 (2000) 485-488.

10) V. A. Chernenko and V. V. Kokorin: Proc. of ICOMAT'92, ed. by C. M. Wayman and J. Perkins, Monterey, CA: Inst. for Adv. Studies (1992) 1205-1210.

11) V. A. Chernenko, V. A. L'vov, M. Pasquale, S. Besseghini, C. Sasso and D. A. Polenur: Int. J. Appl. Electromagnetics and Mechanics 12 (2000) 3-8.

12) R. D. James and M. Wuttig: Philos. Mag. A 77 (1998) 1273-1288.

13) V. A. L'vov, V. A. Chernenko, E. Cesari and J. Pons: Recent Res. Devel. Mater. Sci. Eng. 1 (2002) 673-733.

14) V. A. L'vov and V. A. Chernenko: Eur. Phys. J. AP 8 (1999) 25-28.

15) J. Kim, F. Inaba, T. Fukuda and T. Kakeshita: Acta Mater. 54 (2006) 439-499. 